MIDPI sciforum
MOL2NET, International Conference on Multidisciplinary Sciences, $5^{\text {th }}$ Edition

USINEWS-03: US-IN-EU Worldwide Science Workshop Series, UMN, Duluth, USA, 2019

\title{
Carbonic Anhydrases and their Physiological Roles
}

\author{
Tanya Agarwal, Rajeev K Singla *, Arun Garg \\ Drug Design and Discovery Laboratory, School of Medical and Allied Sciences, KR Mangalam \\ University, Sohna Road, Gurugram-122103, India
}

*Address for Correspondence: Rajeev K Singla, rajeevsingla26@gmail.com

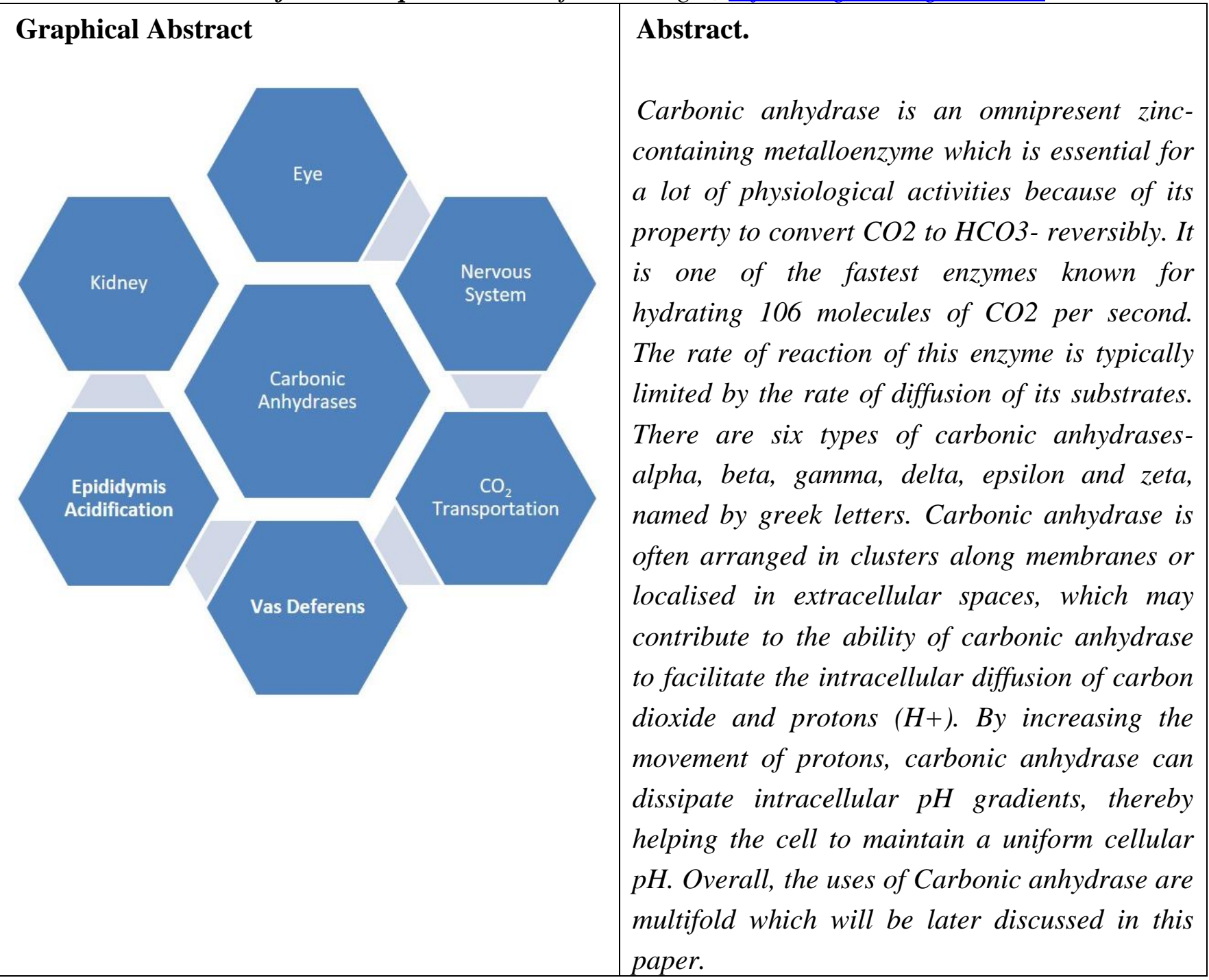

\section{Introduction}

Carbonic anhydrase (EC-4.2.1.1), also known as carbonate dehydratases (Almanza, 2012), is a zinc containing metalloenzyme that catalyses the inter-conversion of dissolved bicarbonates and carbon dioxide. This enzyme was first identified in 1933, in red blood cells of cows (Dutta, 2004) by Meldrum and Roughton in 1933 and Neish accidentally discovered the first $\beta$ carbonic anhydrase as a component of the chloroplast of 
the plant leaf in 1939 (Almanza, 2012). Carbonic anhydrase is one of the fastest enzymes known for hydrating 106 molecules of $\mathrm{CO}_{2}$ per second. The rate of reaction of this enzyme is typically limited by the rate of diffusion of its substrates (Bhat et al, 2017). The carbonic anhydrase reaction is involved in many physiological and pathological processes, including respiration and transport of $\mathrm{CO}_{2}$ and bicarbonate between metabolizing tissues and lungs, $\mathrm{pH}$ and $\mathrm{CO}_{2}$ homeostasis, electrolyte secretion in various tissues and organs, biosynthetic reactions (such as gluconeogenesis, lipogenesis and ureagenesis), bone resorption and calcification (Supuran, 2018). Species can produce many different carbonic anhydrase isozymes, some of which act in the cytosol, while others are membrane-bound. For instance, in humans there are five cytosolic isozymes (I, II, III, VII and XIII) (Supuran, 2018), five membrane-bound isozymes (IV, VII, IX, XII and XIV), two mitochondrial isozymes (VA and $\mathrm{VB}$ ), and a secreted salivary isozyme (VI), as well as several related proteins that lack catalytic activity. Carbonic anhydrases are often arranged in clusters along membranes or localised in extracellular spaces, which may contribute to the ability of carbonic anhydrase to facilitate the intracellular diffusion of carbon dioxide and protons $\left(\mathrm{H}^{+}\right)$. By increasing the movement of protons, carbonic anhydrase can dissipate intracellular $\mathrm{pH}$ gradients, thereby helping the cell to maintain a uniform cellular $\mathrm{pH}$. Carbonic anhydrases can also create localised gradients, which may aid in processes such as facilitated diffusion across a membrane.

\section{Types of Carbonic Anhydrase}

There is a wide variety of carbonic anhydrase proteins that fall into a number of protein families. These families are named by Greek letters and roughly follow the order in which they were discovered (DiMario et al, 2018). The first class, the $\alpha$-CA, was discovered in the 1930 's in vertebrates. The second class, the betaCA, was discovered in the 1990's in plants (DiMario et al, 2018). A third group was identified in archaebacteria in 1994. The latest CA families, the $\eta$-CAs and the $\theta$-CAs, were discovered in 2015 and 2016 respectively. Each protein family is phylogenetically unrelated to the others (DiMario et al, 2018).

\section{ALPHA TYPE}

The $\alpha$-class is found throughout the animal kingdom, in the periplasm (Bhat et al, 2017). The a-carbonic anhydrases are widely identified in vertebrates, algae and in eubacteria (Bhat et al, 2017). They were the first class of carbonic anhydrases which were isolated from erythrocytes. They are distinct from all other carbonic anhydrase classes in both protein structure and amino acid sequence (DiMario et al, 2018). The protein structure includes a central $\beta$-sheet consisting of ten $\beta$-strands surrounded by seven peripheral $\alpha$-helices. The $\beta$-sheet acts as the active site of carbonic anhydrase. It coordinates the zinc atom with three histidine residues and a water molecule (DiMario et al, 2018).

The CA enzymes found in mammals are divided into four broad subgroups, which, in turn consist of several isoforms:

- The cytosolic CAs (CA-I, CA-II, CA-III, CAVII and CA XIII)

- Mitochondrial CAs (CA-VA and CA-VB)

- secreted CAs (CA-VI)

- Membrane-associated CAs (CA-IV, CA-IX, CA-XII, CA-XIV and CA-XV)

There are three additional acatalytic CA isoforms (CA-VIII, CA-X, and CA-XI) whose functions are unclear (Hunter, 2018). 


\subsection{CARBONIC ANHYDRASE I (CAI)}

Carbonic anhydrase I belongs to $\alpha$-CA subfamily and is localized in the cytosol of red blood cell, GI tract, cardiac tissues and other organs or tissues (Cedar101, 2018). The human CAI protein contains an $\mathrm{N}$-terminus active site, zinc binding site, and substrate-binding site. The crystal structure of the human CA1-bicarbonate anion complex has two H-bonds between the Glu106-Thr199 pair and the Glu117-His119 pair, and one pi $\mathrm{H}$-bond between a water molecule and the phenyl ring of the Tyr114 residue (Cedar101, 2018). The catalytic rate of CAI is only about $10 \%$ that of CAII (Cedar101, 2018). It is used in reversible hydration of carbon dioxide and it can hydrate cyanamide to urea (Anonymous, 2018). In a human zinc-activated variant of CA1, the Michigan Variant, a single point mutation changes His 67 to Arg in a critical region of the active site. This variant of the zinc metalloenzyme appears to be unique in that it possesses esterase activity that is specifically enhanced by added free zinc ions (Cedar101, 2018). CAI is activated by histamine, imidazole, L-adrenaline, L and D-histidine, and L and Dphenylalanine (Anonymous, 2018).

\subsection{CARBONIC ANHYDRASE II (CAII)}

Human carbonic anhydrase II is one of the most efficient one in carbonic anhydrase isozymes, which catalyzes the reversible hydration/dehydration of $\mathrm{CO}_{2}$ and water (Anonymous, 2013). It is widely found in human's kidney, brain, Pancreas, gastric mucosa, skeletal muscle, retina and lens and other organizations organs (Anonymous, 2013). It is involved in many pathological and physiological processes such as acid-base balance, cancer, osteoporosis, glaucoma (Anonymous, 2013), regulation of fluid secretion into the anterior chamber of the eye (Anonymous, 2018). It is activated by X-ray, histamine, L-adrenaline, L and D-phenylalanine, L and D-histidine, L-HisOMe and beta-Ala-His (carnosine) (Anonymous, 2018) and competitively inhibited by saccharin, thioxolone, coumarins, 667-coumate, celecoxib (Celebrex), valdecoxib (Bextra) etc (Anonymous, 2018). CAII deficiency produces osteoporosis, renal tubular acidosis and cerebral calcification (Mutat and GN, 2004). Other features include developmental delay, short stature, cognitive defects, and a history of multiple fractures by adolescence (Mutat and GN, 2004).

\subsection{CARBONIC ANHYDRASE III (CAIII)}

Carbonic anhydrase III (CA III) is a cytosolic enzyme which is known to be highly expressed in the skeletal muscle, (Harju et al, 2013), tissues that synthesize and/or store fat: liver, white adipose tissue and brown adipose tissue (Renner, 2017). It is nutritionally regulated at both the mRNA and protein level (Renner, 2017). It helps in reversible hydration of carbon dioxide (Anonymous, 2018). It is activated by proton donors such as imidazole and the dipeptide histidyl-histidine and inhibited by coumarins and sulfonamide derivatives such as acetazolamide (Anonymous, 2018).

\subsection{CARBONIC ANHYDRASE IV (CAIV)}

CA IV is a glycosylphosphatidyl-inositolanchored membrane isozyme expressed on the luminal surfaces of pulmonary (and certain other) capillaries and of proximal renal tubules (Bot, 2018). It is identified in pulmonary epithelium of many mammalian species and may be uniquely adaptive for gas exchange necessary for the high metabolic requirements of mammals (Bot, 2018). It is essential for acid overload removal from the retina and retina epithelium, and acid release in the choriocapillaris in the choroid (Anonymous, 2018). Carbonic anhydrase IV is activated by histamine, L-adrenaline, Dphenylalanine, L and D-histidine and inhibited by coumarins, saccharin, sulfonamide derivatives such as acetazolamide and Foscarnet (Anonymous, 2018).

\subsection{CARBONIC ANHYDRASE V (CAV)}

Carbonic anhydrase V is of two types CAVA and CAVB. CAVB is localized in the mitochondria and shows the highest sequence similarity to the other mitochondrial CA, CAVA. It has a wider tissue distribution than CAVA (Anonymous, 2018). The carbonic anhydrase VA enzyme is particularly important in the liver, where it provides bicarbonate needed by four enzymes in the energy-producing centers of cells 
DOI : $10.3390 / \mathrm{mol}$ net-05-xxxxx

(mitochondria): carbomoyl phosphate synthetase1, pyruvate carboxylase, propionyl-CoA carboxylase, and 3-methylcrotonyl-CoA carboxylase.

The differences in tissue distribution suggest that the two mitochondrial carbonic anhydrases evolved to assume different physiologic roles.

\subsection{CARBONIC ANHYDRASE VI (CAVI)}

Carbonic anhydrase VI (CA VI) is a secretory isozyme of the $\alpha$-CA gene family (Patrikainen et al, 2014) and it was initially described in the ovine parotid gland and saliva (Kivela et al, 1997). It is highly expressed in the salivary and mammary glands and secreted into saliva and milk (Patrikainen et al, 2014) and because of its presence in saliva it is known as 'gustin' (Rjwilmsi, 2018).

\subsection{CARBONIC ANHYDRASE VII (CAVII)}

Human carbonic anhydrase VII (CA VII) is a cytosolic isoform belonging to the $\alpha$-CA family that shows high carbon dioxide hydration activity (Monti et al, 2015). It is a protein coding gene (Anonymous, 2018) and is present in saliva (KolbertBot, 2017). It acts as oxygen radical scavenger and helps in $\mathrm{pH}$ regulation. It is involved in the initial postnatal phases of brain development, as well as in the generation of neuronal excitation and febrile seizures (Monti et al, 2015). Diseases associated with CAVII include Trichosporonosis (Anonymous, 2018). It is activated by histamine, $\mathrm{L}$-adrenaline, $\mathrm{L}$ and $\mathrm{D}$ histidine, and $\mathrm{L}$ and D-phenylalanine and inhibited by coumarins, sulfonamide derivatives such as acetazolamide (AZA), by saccharin and Foscarnet (Anonymous, 2018).

\subsection{CARBONIC ANHYDRASE VIII} (CAVIII)

The protein encoded by this gene was initially named CA-related protein because of sequence similarity to other known carbonic anhydrase genes. However, the gene product lacks carbonic anhydrase activity (i.e., the reversible hydration of carbon dioxide). The gene product continues to carry a carbonic anhydrase designation based on clear sequence identity to other members of the carbonic anhydrase gene family (KolbertBot,
2017). Mutations in this gene are associated with cerebellar ataxia, mental retardation, and dysequilibrium syndrome 3 (CMARQ3). Polymorphisms in this gene are associated with osteoporosis, and overexpression of this gene in osteosarcoma cells suggests an oncogenic role (Anonymous, 2018).

\subsection{CARBONIC ANHYDRASE IX (CAIX)}

CAIX is a transmembrane protein and is a tumorassociated carbonic anhydrase isoenzyme. It is over-expressed in VHL mutated clear-cell renal cell carcinoma (ccRCC) and hypoxic solid tumors, but is low-expressed in normal kidney and most other normal tissues (OAbot, 2018). The expression of CAIX is upregulated during hypoxic conditions (Thakur and Enwere, 2016). It may be involved in cell proliferation and transformation (OAbot, 2018).

\subsection{CARBONIC ANHYDRASE X (CAX)}

The protein encoded by this gene is an acatalytic member of the alpha-carbonic anhydrase subgroup, and it is thought to play a role in the central nervous system, especially in brain development (Anonymous, 2018). It does not have any catalytic activity (Anonymous, 2018).

\subsection{CARBONIC ANHYDRASE XI (CAXI)}

CA XI is likely a secreted protein, but radical changes at active site residues completely conserved in CA isozymes with catalytic activity, make it unlikely that it has carbonic anhydrase activity. It shares properties in common with two other acatalytic CA isoforms, CA VIII and CA $\mathrm{X}$. It is expressed abundantly in the brain with moderate expression also present in spinal cord and thyroid (Anonymous, 2018).

\subsection{CARBONIC ANHYDRASE XII (CAXII)}

CAXII is a type-I membrane protein (Anonymous, 2018) that is highly expressed in colon, kidney, prostate, intestine and activated lymphocytes, expressed at much higher levels in the renal cell cancers than in surrounding normal kidney tissue and moderately expressed in pancreas, ovary and testis (Anonymous, 2018). Expression of CAXII is regulated by hypoxia and estrogen receptors (Waheed and Sly, 2017). 


\subsection{CARBONIC ANHYDRASE XIII (CAXIII)}

CA13 (Carbonic Anhydrase 13) is a protein coding gene. Among its related pathways are Metabolism and Nitrogen metabolism. Gene Ontology (GO) annotations related to this gene include carbonate dehydratase activity (Anonymous, 2018). It is expressed in thymus, small intestine, spleen, prostate, ovary, colon and testis (Anonymous, 2018).

\subsection{CARBONIC ANHYDRASE XIV (CAXIV)}

CA XIV is predicted to be a type I membrane protein and shares highest sequence similarity with the other trans-membrane CA isoform, CA XII; however, they have different patterns of tissue-specific expression and thus may play different physiologic roles (Anonymous, 2018). It is highly expressed in all parts of the central nervous system and less expressed in adult liver, heart, small intestine, colon, kidney, urinary bladder and skeletal muscle (Anonymous, 2018).

\subsection{CARBONIC ANHYDRASE XV (CAXV)}

Carbonic anhydrase $\mathrm{XV}$ is a membrane associated a-carbonic anhydrase. It is repressed by coumarins (Anonymous, 2018).

\section{BETA TYPE}

The $\beta$-carbonic anhydrases are mostly found in higher plants, micro-algae, Eubacteria, Archaebacteria, Cyanobacteria and Fungi (Bhat et al, 2017). It is an essential component of carboxyzome, an organelle with high $\mathrm{CO}_{2}$ concentrations in cyanobacteria. In aerobic prokaryotes, beta-class CAs are implicated in maintaining internal $\mathrm{pH}$ and $\mathrm{CO}_{2} /$ bicarbonate balances required for biosynthetic reactions (Zimmerman and Ferry, 2008). In anaerobic prokaryotes, beta-class CAs are implicated in the transport of $\mathrm{CO}_{2}$ and bicarbonate across the cytoplasmic membrane that regulates $\mathrm{pH}$ and facilitates acquisition of substrates and product removal required for growth. In phototrophic organisms, beta-class CAs are particularly important for transport and concentration of $\mathrm{CO}_{2}$ and bicarbonate for photosynthesis (Zimmerman and Ferry, 2008).

\section{GAMMA TYPE}

The $\gamma$-carbonic anhydrases are present in algae (Bhat et al, 2017), plants, photosynthetic bacteria.

The $\gamma$-carbonic anhydrases were first discovered in the Archaeon Methanosarcina thermophile. Physiological roles for the gamma-class are not as well documented (Zimmerman and Ferry, 2008). The archaebacterial $\gamma$-CA possesses a zinc active site coordinated by three histidine residues and one water molecule. To form the $\gamma \mathrm{CA}$ active site, one monomer provides two zinccoordinating histidine residues whereas a second monomer provides the third zinc-coordinating histidine residue (DiMario et al, 2018). Altogether, three monomers interact to form three active sites per homotrimer, the active $\gamma$ $\mathrm{CA}$ unit. One of the cyanobacterial ptotein, $\mathrm{n}$ $\mathrm{CcmM}$ is also a $\gamma$-CA. The $\mathrm{N}$-terminus of $\mathrm{CcmM}$ has a $\gamma$-CA domain while the $\mathrm{C}$-terminus has three to four $\mathrm{RbcS}$ domains allowing the protein to coordinate Rubisco packaging in the carboxysome (DiMario et al, 2018).

\section{DELTA TYPE}

The $\delta$-carbonic anhydrases have only been described in some diatoms. The $\delta$-carbonic anhydrase families appear to be a case of convergent evolution with almost no sequence similarity with the $a, \beta$ or $\gamma$ carbonic anhydrase types (Bhat et al, 2017). It is proposed to function in marine diatoms to concentrate $\mathrm{CO}_{2}$ for photosynthesis (Zimmerman and Ferry, 2008). The $\delta$-CA, TWCA1 was reported in 1997 (DiMario et al, 2018).

\section{EPSILON TYPE}

$\varepsilon$-carbonic anhydrase is a part of the carboxysome shell and has additional domains that serve the function in bacteria (Bhat et al, 2017). They are found in cyanobacteria and are now recognized as being a highly modified $\beta$ Carbonic Anhydrase (DiMario et al, 2018). 


\section{ZETA TYPE}

$\zeta$-carbonic anhydrase is limited to maline protists and resembles the $\beta$-carbonic anhydrase family, with other metals such as $\mathrm{Cd}$ or Co substitute for $\mathrm{Zn}$ (Bhat et al, 2017). The $\zeta \mathrm{CA}, \mathrm{CDCA} 1$ was reported a few years earlier. The $\mathrm{Zn}$-binding region of CDCA1 is repeated three times and its structure was reported in 2012 (DiMario et al, 2018). An unusual aspect of the CDCA1 is its ability to use other metals besides Zn. Activity has been reported for this $\zeta$-CA when binding others metals, most notably Cd (DiMario et al, 2018). This ability to bind other metals might be an evolutionary adaptation to the low $\mathrm{Zn}$ levels often found in oceanic environments.

\section{Mechanism}

The carbonic anhydrase active sites contain a zinc ion and all share a similar catalytic activity. In the carbonic anhydrase enzyme, a zinc prosthetic group is coordinated in three positions by histidine side-chains. The active site of the enzyme "E" contains a specific pocket for $\mathrm{CO}_{2}$ which brings it closer to the hydroxide group attached to the zinc. Therefore, this causes the electron-rich hydroxide ion to attack the $\mathrm{CO}_{2}$ thereby creating a bicarbonate molecule.

$\mathrm{CO}_{2}+\mathrm{H}_{2} \mathrm{O} \leftrightarrow \mathrm{HCO}_{3}{ }^{-}+\mathrm{H}^{+}$

\section{Physiological Roles}

Carbonic anhydrase plays a key role in the regulation of $\mathrm{pH}$ and fluid balance in different parts of the body (Dutta, 2004). Bicarbonate, a primary substrate of carbonic anhydrase, is active in many biological processes: (i) as a counter-ion in sodium transport, (ii) as a carrier for $\mathrm{CO}_{2}$, (iii) as a buffer, and (iv)as a metabolite in biosynthetic reaction pathways.

In mammals, carbonic anhydrase catalyzes the reversible hydration of $\mathrm{CO}_{2}$ to bicarbonate (and the reverse reaction). These reactions are important to a variety of biological processes, including the following: (i) regulation of respiration and gas exchange, (ii) regulation of acid-base equilibria, (iii) vision, (iv) development and function of bone, (v) calcification, (vi) metabolism, (vii) signalling and memory, (viii) gustation,(ix) production of saliva, (x) production of pancreatic juices, (xi) intestinal transport of ions, (xii) muscle function and the nervous system, (xiii) regulation of seminal fluid, (xiv) adaptation to cellular stress, (xv) acidification of the extracellular environment around hypoxic tumour cells, and (xvi) several biosynthetic pathways.

\section{ROLE OF CARBONIC ANHYDRASE IN EYE}

Carbonic anhydrase plays an important role in the eye, where it is present in the lens, vitreous body, cornea, and retina. Within the ciliary body, the CA II-catalyzed formation of bicarbonate is the primary mechanism for the transport of sodium into the eye. The influx of sodium ions into the eye is accompanied by the transport of water, both processes are important in maintaining the aqueous humor. Inhibition of carbonic anhydrase decreases the production of bicarbonate, which subsequently lowers intraocular pressure.

\section{NERVOUS SYSTEM}

In the nervous system, carbonic anhydrase serves many functions. In the choroid plexus, it contributes to the production of cerebrospinal fluid. In the brain, it is found in oligodendrocytes and glial cells but is at the highest concentrations in sensory neurons, where it is important in signal processing, long-term synaptic transformation, and attentional gating of memory storage. Activation of carbonic anhydrase rapidly increases levels of bicarbonate in memory-related neural structures. Regulation of the flux of bicarbonate into synaptic receptor channels allows carbonic anhydrase to function as a gate that regulates the transfer of signals through the neural network.

\section{3. $\mathrm{CO}_{2}$ TRANSPORT IN BLOOD AND MUSCLES}

$\mathrm{CO}_{2}$ produced within skeletal muscle has to leave the body finally via ventilation by the lungs. To get there, $\mathrm{CO}_{2}$ diffuses from the intracellular space into the connective transport medium, blood with the two compartments, plasma and erythrocytes (Geers and Gros, 2000). Within the body, $\mathrm{CO}_{2}$ is transported in three different forms: physically dissolved, as $\mathrm{HCO}_{3}{ }^{-}$, or as carbamate. 
The relative contribution of these three forms to overall transport is changes along the elimination pathway. Carbonic anhydrase accelerates the hydration/dehydration reaction between $\mathrm{CO}_{2}$, $\mathrm{HCO}_{3}{ }^{-}$, and $\mathrm{H}^{+}$. In skeletal muscle, various isozymes of carbonic anhydrase are localized within erythrocytes but are also bound to the capillary wall, thus accessible to plasma; bound to the sarcolemma, thus producing catalytic activity within the interstitial space; and associated with the sarcoplasmic reticulum. In some fibre types, carbonic anhydrase is also present in the sarcoplasm (Geers and Gros, 2000). In exercising skeletal muscle, lactic acid contributes huge amounts of $\mathrm{H}^{+}$and by these affects the relative contribution of the three forms of $\mathrm{CO}_{2}$.

\section{VAS DEFERENS AND EPIDIDYMIS ACIDIFICATION}

CA II is involved in net proton secretion by clear cells in the distal portion of the epididymis and the vas deferens and CA IV participates in bicarbonate reabsorption by principal cells of the proximal regions (Breton, 2001).

In order to maintain sperm in inactive state during maturation and storage a low bicarbonate concentration and an acidic $\mathrm{pH}$ in the luminal fluid of the epididymis and vas deferens is required. Active bicarbonate reabsorption takes place in the proximal regions of epididymis and net proton secretion occurs in the distal portions of the epididymis (cauda epididymidis) and vas deferens (Breton, 2001). Narrow and clear cells of epithelium lining the epididymis and vas deferens express high levels of the vacuolar type $\mathrm{H}^{+}$ATPase on their apical membrane and subapical vesicles, and high levels of cytosolic CA II, indicating that these cells are involved in proton secretion coupled to bicarbonate reabsorption. Epididymal principal cells also show apical carbonic anhydrase activity and CA IV is expressed in their apical membrane (Breton, 2001).

\section{KIDNEY}

Carbonic anhydrase is expressed in most segments of the kidney. CAII and CAIV are predominate in human and rabbit kidneys, CAXII, and CAXIV are also present. CAIX is expressed by renal cell carcinoma (RCC) (Purkerson and Schwartz, 2007). In the kidney, this enzyme is thought to play a pivotal role in urinary acidification and bicarbonate reabsorption (Dobyan and Bulger, 1982). Acidification of urine could be brought about either by the secretion of hydrogen ions into the tubular fluid or by the selective absorption of a buffer base. Both filtration and secretion are essential to hydrogen ion excretion and both proximal and distal convoluted tubules are involved.

The bulk of the bicarbonate filtered at the glomerulus is reabsorbed in the proximal tubule, from which it passes back into the peritubular capillaries. This mechanism is designed to keep the normal plasma bicarbonate concentration constant at about 25 millimoles per litre. When the plasma concentration falls below this level, no bicarbonate is excreted and all filtered bicarbonate is reabsorbed into the blood. When the plasma bicarbonate rises above 27 millimoles per litre, bicarbonate appears in the urine in increasing amounts (Dobyan and Bulger, 1982).

The brush borders of the cells of the proximal tubules are rich in the enzyme carbonic anhydrase. This enzyme facilitates the formation of carbonic acid $\left(\mathrm{H}_{2} \mathrm{CO}_{3}\right)$ from $\mathrm{CO}_{2}$ and $\mathrm{H}_{2} \mathrm{O}$, which then ionizes to hydrogen ions $\left(\mathrm{H}^{+}\right)$and bicarbonate ions $\left(\mathrm{HCO}_{3}^{-}\right)$. The starting point for bicarbonate reabsorption is probably the active secretion of hydrogen ions into the tubular fluid. These ions are formed under the influence of carbonic anhydrase from $\mathrm{CO}_{2}$ liberated from oxidation of cell nutrients and $\mathrm{H}_{2} \mathrm{O}$ already in the cells. The filtered base, bicarbonate, accepts the hydrogen ions to form carbonic acid, which is unstable and dissociates to form $\mathrm{CO}_{2}$ and $\mathrm{H}_{2} \mathrm{O}$. The partial pressure of $\mathrm{CO}_{2}$ in the filtrate rises, and, as $\mathrm{CO}_{2}$ is highly diffusible, it passes readily from the tubular fluid into the tubular cells and the blood, and the water is either dealt with in the same way or is excreted. In the meantime the proximal tubular cells are actively reabsorbing filtered sodium, which is balanced by the $\mathrm{HCO}_{3}{ }^{-}$ formed within the cells from the $\mathrm{CO}_{2}$ generated by the hydrogen ions in the luminal fluid (Dobyan and Bulger, 1982). Thus the bicarbonate actually reabsorbed is not that which was originally the filtrate, but the net effect is the same as if this was the case. 
Thus, Carbonic anhydrase isozymes perform different functions at their specific locations, and their absence or malfunction can lead to diseased states, ranging from the loss of acid production in the stomach to kidney failure.

\section{Conclusion}

The carbonic anhydrases (or carbonate dehydratases) form a family of enzymes that catalyse the inter-conversion between carbon dioxide and water andthe dissociated ions of carbonic acid (i.e. bicarbonate and protons). This inter-conversion is a reversible reaction and the enzyme catalyses both reactions, forward and reverse. The active site of most carbonic anhydrases contains a zinc ion; they are therefore classified as metalloenzymes. There are currently six (alpha, beta, gamma, delta, zeta and epsilon) classes of carbonic anhydrases (CAs) of which the alpha-class from mammalian sources has been studied to a much greater extent compared to the other classes. This enzyme is found in many tissues such as the gastrointestinal tract $(\mathrm{GI})$, the reproductive tract, the nervous system, kidneys, lungs, skin and eyes, wherein it plays key roles in a number of physiological and pathological processes, such as $\mathrm{pH}$ regulation, ions and gas exchanges, calcification, photosynthesis, tonic modulation of brain excitability through modulation of amino acid receptors, and biosynthetic reactions (such as gluconeogenesis, lipogenesis, and ureagenesis). Carbonic anhydrase isozymes perform different functions at their specific locations, and their absence or malfunction can lead to diseased states, ranging from the loss of acid production in the stomach to kidney failure. Overall, the uses of carbonic anhydrase are multifold and hence carbonic anhydrase is an important enzyme.

\section{References}

1. Bhat, F.A; Ganai, B.A; Uqab, B, Carbonic anhydrase: Mechanism, Structure and Importance in Higher Plants, Asian journal of plant science and research, 2017, 7(3),1723

2. Supuran, C.T, Carbonic Anhydrases as targets for medicinal chemistry, Bioorganic and medicinal chemistry, 2007, 15(13), 4336-4350
3. Breton, S, The Cellular Physiology of Carbonic Anhydrases, JOP.J.Pancreas, 2001, 2(4), 159-164

4. Supuran, C.T, Carbonic Anhydrases: novel therapeutic applications for inhibitors and activators, Nature Publishing Group, 2008, 7, 168-181

5. DiMario, R.J; Machingura, M.C; Waldrop, G.L; Moroney, J.V, The many types of carbonic anhydrases in photosynthetic organisms, Plant science,2018, 268,11-17

6. Zimmerman, S.A; Ferry, J.G, The beta and gamma classes of carbonic anhydrase, Current Pharmaceutical Design, 2008, 14(7),716-721

7. Dutta, S; Goodsell, D.S, pdb101.rcsb.org, url: https://pdb101.rcsb.org/motm/49, $15^{\text {th }}$ September, 2018

8. Auerbach S, sciencing.com, url: https://sciencing.com/functions-carbonicanhydrase-7426.html, $17^{\text {th }}$ September 2018

9. Hunter, Adrian J, wikipedia.org, url: https://en.wikipedia.org/wiki/Carbonic anhy drase\#\%CE\%B1-CA,

$2018,18^{\text {th }}$

September,2018

10. Coleman,J.R, link.springer.com,url: https://link.springer.com/chapter/10.1007\%2 F0-306-48137-5_15, $18^{\text {th }}$ September,2018

11. Boone, christopher D; Gill, sonika; Habibzadegan, Andrew; McKenna, Robert, Carbonic anhydrase:a An efficient enzyme with possible global implications, International journal of chemical engineering, 2013

12. Abdoli, Morteza; Angeli, Andrea; Bozdag, Murat; Carta, Fabrizio; Kakanejadifard, Ali; Saeidian, Hamid; Supuran, Claudiu T, Synthesis and carbonic anhydrase I, II, VII and IX inhibition studies with a series of benzo[d]thiazole-5-and-6- sulfonamides, Journal of enzyme inhibition and medicinal chemistry, 32(1), 2017, 1071-1078

13. Vinoth, M; Rani, G Jhasi; Anitha, P, Docking studies on novel human carbonic anhydrase II, International journal of biomedical and advance research, 2(10), 2011, 410-415 
DOI : 10.3390/mol2net-05-xxxxx

14. Geers, Cornelia; Gros,

Gerolf, physiology.org,

url:https://www.physiology.org/doi/full/10.1

$152 /$ physrev.2000.80.2.681 $, \quad 2000, \quad 17^{\text {th }}$ October,2018

15. Almanza, Dalila jacqueline escudero; Barrios, Damaris leopoldina ojeda; Rodriguez, Ofelia adriana hernandez; Chavez, Esteban sanchez; Anchondo, Teresita ruiz; Arreola, Juan pedro sita, Carbonic anhydrase and zinc in plant physiology, Chilean journal of agricultural research, 72(1), 2012, 140-146

16. McDowall, Jennifer, ebi.ac.uk, url:https://www.ebi.ac.uk/interpro/potm/200 4 1/Page1.htm, $19^{\text {th }}$ October, 2018

17. Dobyan, D.C; Bulger, R.E, physiology.org, url:

https://www.physiology.org/doi/pdf/10.1152/ ajprenal.1982.243.4.F311, $\quad 1982, \quad 21^{\text {st }}$ October, 2018

18. Cedar101, wikipedia.org, url:https://en.m.wikipedia.org/wiki/CA1_(ge ne), 2018, $18^{\text {th }}$ October, 2018

19. Anonymous, uniprot.org, url:https://www.uniprot.org/uniprot/P00915, $18^{\text {th }}$ October, 2018

20. Anonymous, chem.libretexts.org, url:https://chem.libretexts.org/LibreTexts/Un iversity_of_California_Davis/UCD_Chem_1 24A\%3A_Berben/Carbonic_Anhydrase/Carb onic Anhydrase 1 1\#title, 2013, $18^{\text {th }}$ October, 2018

21. Anonymous, uniprot.org,

url:https://www.uniprot.org/uniprot/P00918, $18^{\text {th }}$ October, 2018

22. GN, Shah; Mutat, Hum, Carbonic anhydrase II deficiency syndrome (osteopetrosis with renal tubular acidosis and brain calcification): novel mutations in CA2 identified by direct sequencing expand the opportunity for genotype-phenotype correlation, 24(3), 2004, 272

23. Bot, Bibcode, wikipedia.org, url:https://en.m.wikipedia.org/wiki/Carbonic anhydrase III, 2018, 19 ${ }^{\text {th }}$ October, 2018

24. Anonymous, ncbi.nlm.nih, url:https://www.ncbi.nlm.nih.gov/gene/761, 2018, 22 $2^{\text {nd }}$ October, 2018

25. Harju, Anna-Kaisa; Bootorabi, Fatemeh; Kuualahti, Marianne; Supuran, Claudiu T; Parkkila, Seppo, Carbonic anhydrase III: A neglected isozyme is stepping into the limelight, Journal of enzyme inhibition and Medicinal chemistry, 28(2), 2013, 231-239

26. Anonymous, uniprot.org, url:https://www.uniprot.org/uniprot/P07451, $20^{\text {th }}$ October, 2018

27. Renner, Sarah W; Walker, Lauren M; Forsberg, Lawrence J; Sexton, Jonathan Z; Brenman, Jay E, Carbonic anhydrase III (Car3) is not required for fatty acid synthesis and does not protect against high-fat diet induced obesity in mice, PLOS one, 12(4), 2017

28. Fiore, Anna Di; Monti, Daria M; Scaloni, Andrea; Simone, Giuseppina De; Monti, Simona M, Protective Role of Carbonic Anhydrases III and VII in Cellular Defense Mechanisms upon Redox Unbalance, Oxidative medicine and cellular longevity, 2018

29. Wandernoth, Petra M; Raubuch, Michael; Mannowetz, Nadja; Becker, Holger M; Deitmer, Joachim W; Sly, William S; Wennemuth, Gunther, Role of Carbonic Anhydrase IV in the Bicarbonate-Mediated Activation of Murine and Human Sperm, PLOS one, 5(11), 2010

30. Anonymous, uniprot.org, url:https://www.uniprot.org/uniprot/P22748, $22^{\text {nd }}$ October, 2018

31. Bot, Kolbert, wikipedia.org, url:https://en.m.wikipedia.org/wiki/Carbonic anhydrase 4 , $20^{\text {th }}$ October,2018

32. Anonymous, uniprot.org, url:https://www.uniprot.org/uniprot/Q10462, $22^{\text {nd }}$ October, 2018

33. Patrikainen, Maarit; Pan, Peiwen; Kulesskaya, Natalia; Voikar, Vootele; Parkkila, Seppo, The role of carbonic anhydrase VI in bitter taste perception: evidence from the Car6-/- mouse model, Journal of biomedical science, 21(1), 2014, 82

34. Kivela, Jyrki; Parkkila, Seppo; Waheed, Abdul; Parkkila, Anna-Kaisa; Sly, William S; Rajaniemi, Hannu, Secretory carbonic anhydrase isoenzyme (CA VI) in human serum, Clinical chemistry, 43(12), 1997, 2318-2322

35. Rjwilmsi, wikipedia.org, url:https://en.m.wikipedia.org/wiki/Carbonic _anhydrase $\_$VI, 2018, 22 $2^{\text {nd }}$ October, 2018 
DOI : 10.3390/mol2net-05-xxxxx

36. KolbeBot, wikipedia.org, url:https://en.m.wikipedia.org/wiki/Carbonic _anhydrase 7 , 2017, 22 ${ }^{\text {nd }}$ October, 2018

37. Anonymous, uniprot.org, url:https://www.uniprot.org/uniprot/P43166, $22^{\text {nd }}$ October, 2018

38. Anonymous, genecards.org, url:https://www.genecards.org/cgibin/carddisp.pl?gene $=\mathrm{CA} 7, \quad 21^{\text {st }}$ October, 2018

39. Monti, Simona M; Supuran, Claudiu T; Simone, Giuseppina De; Fiore, Anna Di, Carbonic anhydrases as biocatalysts, Theory to medical and industrial applications, 2015, 151-168

40. KolberBot, wikipedia.org, url:https://en.m.wikipedia.org/wiki/CA8, 2017, $22^{\text {nd }}$ October, 2018

41. Anonymous, genecards.org, url:https://www.genecards.org/cgibin/carddisp.pl?gene $=\mathrm{CA} 8,22^{\text {nd }}$ October, 2018

42. OAbot, wikipedia.org, url:https://en.m.wikipedia.org/wiki/Carbonic anhydrase_9 $, 2018,20^{\text {th }}$ October, 2018

43. Thakur, Satbir; Enwere, Emeka, pathologyoutlines.com, url: http://www.pathologyoutlines.com/topic/stai nscarbonicanhydraseix.html, $2016, \quad 19^{\text {th }}$ October, 2018

44. Anonymous, genecards.org, url:https://www.genecards.org/cgibin/carddisp.pl?gene $=$ CA10, $19^{\text {th }}$ October, 2018

45. Anonymous, uniprot.org, url:https://www.uniprot.org/uniprot/Q9NS85 , $19^{\text {th }}$ October, 2018

46. Anonymous, Wikipedia.org, url: https://en.m.wikipedia.org/wiki/CA11,$\quad 19^{\text {th }}$ October, 2018

47. Anonymous, genecards.org, url: https://www.genecards.org/cgibin/carddisp.pl?gene $=$ CA11, $20^{\text {th }}$ October, 2018

48. Anonymous, uniprot.org, url: https://www.uniprot.org/uniprot/O75493, $20^{\text {th }}$ October, 2018

49. Anonymous, genecards.org, url: https://www.genecards.org/cgibin/carddisp.pl?gene $=\mathrm{CA} 12,20^{\text {th }}$ October, 2018
50. OAbot,

Wikipedia.org, url: https://en.m.wikipedia.org/wiki/CA12, 2018, $20^{\text {th }}$ October, 2018

51. Anonymous, uniprot.org, url: https://www.uniprot.org/uniprot/O43570, $19^{\text {th }}$ October, 2018

52. Waheed, Abdul; Sly, William S, Carbonic anhydrase XII functions in health and disease, Elsevier, 623, 2017, 33-40

53. Anonymous, uniprot.org, url: https://www.uniprot.org/uniprot/Q8N1Q1, $19^{\text {th }}$ October, 2018

54. Anonymous, genecards.org, url: https://www.genecards.org/cgibin/carddisp.pl?gene $=$ CA13, $20^{\text {th }}$ October, 2018

55. BoyKolbert, Wikipedia.org, url: https://en.m.wikipedia.org/wiki/CA14, 2017, $19^{\text {th }}$ October, 2018

56. Anonymous, genecards.org, url: https://www.genecards.org/cgibin/carddisp.pl?gene $=$ CA14, $20^{\text {th }}$ October, 2018

57. Anonymous, uniprot.org, url: https://www.uniprot.org/uniprot/Q9ULX7, $20^{\text {th }}$ October, 2018

58. Anonymous, uniprot.org, url: https://www.uniprot.org/uniprot/Q99N23, $20^{\text {th }}$ October, 2018

59. Purkerson, J.M; Schwartz, The role of carbonic anhydrase in renal physiology, Kidney international, 71(2), 2007, 103-115

60. Anonymous, ncbi.nlm.nih.gov, url:https://www.ncbi.nlm.nih.gov/gene/1123 $\underline{8}, 2018,22^{\text {nd }}$ October 2018 